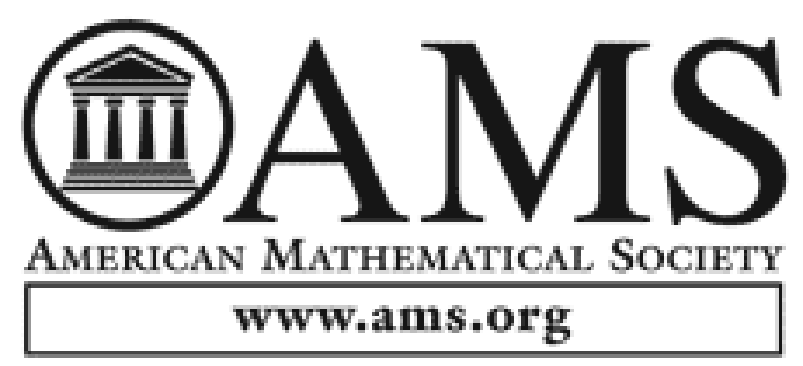

Affine 3-Spheres with Constant Affine Curvature

Author(s): Martin A. Magid and Patrick J. Ryan

Reviewed work(s):

Source: Transactions of the American Mathematical Society, Vol. 330, No. 2 (Apr., 1992), pp.

887-901

Published by: American Mathematical Society

Stable URL: http://www.jstor.org/stable/2153940

Accessed: 05/04/2012 11:43

Your use of the JSTOR archive indicates your acceptance of the Terms \& Conditions of Use, available at http://www.jstor.org/page/info/about/policies/terms.jsp

JSTOR is a not-for-profit service that helps scholars, researchers, and students discover, use, and build upon a wide range of content in a trusted digital archive. We use information technology and tools to increase productivity and facilitate new forms of scholarship. For more information about JSTOR, please contact support@ jstor.org. 


\title{
AFFINE 3-SPHERES WITH CONSTANT AFFINE CURVATURE
}

\author{
MARTIN A. MAGID AND PATRICK J. RYAN
}

\begin{abstract}
We classify the affine hyperspheres in $R^{4}$ which have constant curvature in the affine metric $h$ and whose Pick invariant is nonzero. In particular, the metric $h$ must be flat.
\end{abstract}

\section{INTRODUCTION}

In the affine theory of hypersurfaces, the affine hyperspheres are analogous to the umbilic hypersurfaces in the Riemannian theory. Both are defined by the condition that the shape operator is a constant multiple of the identity. Although the list of umbilic hypersurfaces in real space forms is well known and can be derived as an easy consequence of the Codazzi equation, the situation in affine differential geometry is not so simple.

In fact, there are affine spheres whose defining equations involve arbitrary smooth functions (see, for example, $[5, \S 5]$ ). Thus it is appropriate to use some additional criteria to distinguish various classes of affine hyperspheres.

One approach is to restrict one's attention to so-called locally strongly convex immersions with the result that the affine metric is positive definite. This is a strong assumption and leads to general results. Such an approach has been taken by Cheng and Yau [3] and others.

Our approach is to allow $h$ to be indefinite but to arrive at a specific list of examples characterized in terms of the geometry of $h$. In our previous paper [5], we found the affine 2-spheres for which $h$ is flat and U. Simon [8] has settled the case of nonzero constant curvature. In the present paper we use similar methods, along with the ideas of Yu [9], to study affine 3-spheres in $R^{4}$. We will state our main result in $\S 2$ after some basic terminology has been introduced.

In this paper all maps and manifolds are assumed to be smooth $\left(C^{\infty}\right)$ and all manifolds connected unless otherwise stated. The authors wish to thank Professor K. Nomizu for helpful conversations during the preparation of this paper, and the referee for pointing out a gap in the original proof which led to the introduction of Lemma $5 \mathrm{a}$.

Received by the editors December 18, 1989 and, in revised form, May 18, 1990.

1980 Mathematics Subject Classification (1985 Revision). Primary 53C40; Secondary 53A15, $53 \mathrm{C} 50$.

Key words and phrases. Affine sphere, Pick invariant.

Research of the first author was partially supported by NSF Grant DMS 8802664 .

Research of the second author was partially supported by NSERC Operating Grant A2501. 


\section{AfFine hypersurfaCES IN $R^{n+1}$}

Let $f: M^{n} \rightarrow R^{n+1}$ be an immersion. For any vector field $\xi$ transverse to $M$, we decompose $D_{X} f_{*} Y$ into its tangential and transverse components as follows:

$$
D_{X} f_{*} Y=f_{*}\left(\nabla_{X} Y\right)+h(X, Y) \xi,
$$

where $D$ is the standard flat affine connection on $R^{n+1}$, and $X$ and $Y$ are vector fields tangent to $M$. Nondegeneracy of $h$ is independent of the choice of $\xi$ and we take $h$ to be nondegenerate throughout. $\xi$ determines a volume form $\omega$ by $\omega\left(X_{1}, X_{2}, \ldots, X_{n}\right)=\operatorname{det}\left(X_{1}, X_{2}, \ldots, X_{n}, \xi\right)$; there is a unique choice (up to a sign) of $\xi$ such that $\omega$ coincides with the volume element of the nondegenerate metric $h$ and $D_{X} \xi$ is tangent to $M$ for all $X$ tangent to $M$. Such a choice of $\xi$ (called the affine normal of the immersion) determines a unique induced affine connection $\nabla$ and the corresponding $h$ is called the affine metric. (This metric is sometimes referred to as the Berwald-Blaschke metric.) The shape operator $S$ is defined by

$$
D_{X} \xi=-f_{*}(S X) \text {. }
$$

The difference between the induced connection $\nabla$ and the Levi-Civita connection $\hat{\nabla}$ of the metric $h$ is measured by the tensor $K$ which is defined by

$$
K_{X} Y=\nabla_{X} Y-\hat{\nabla}_{X} Y
$$

for $X$ and $Y$ tangent to $M$.

As in Riemannian theory, (1) and (2) lead to Gauss and Codazzi equations which in this case relate $h, S$, and the curvature tensor $R$ of $\nabla$. The cubic form of the immersion is a symmetric $(0,3)$ tensor $C$ characterized by

$$
C(X, Y, Z)=\left(\nabla_{X} h\right)(Y, Z)=-2 h\left(K_{X} Y, Z\right) .
$$

It is a useful fact (called the apolarity condition) that each $K_{X}$ has zero trace.

The (possibly indefinite) metric $h$ may be extended in a natural way to the algebra of tensor fields over $M$. A particularly important quantity is the Pick invariant $J$ defined by

$$
n(n-1) J=h(K, K)=\frac{1}{4} h(C, C)
$$

and it is straightforward to show that

$$
\operatorname{trace} \widehat{\operatorname{Ric}}=(n-1) \operatorname{trace} S+n(n-1) J,
$$

where $\widehat{\mathrm{Ric}}$ is the Ricci tensor of $h$. Finally, the curvature tensor $\widehat{R}$ of $h$ can be expressed in terms of $h, S$, and $K$ by the formula

$$
\begin{aligned}
\widehat{R}(X, Y) Z=\frac{1}{2}(h(Y, Z) S X-h(X, Z) S Y & \\
& +h(S Y, Z) X-h(S X, Z) Y)-\left[K_{X}, K_{Y}\right] Z .
\end{aligned}
$$

When $S$ is a multiple of the identity, the hypersurface is called an affine hypersphere. In this case we write $S=\lambda I$ and $\lambda$ is automatically constant. If $\lambda$ is nonzero, the affine sphere is called proper; otherwise it is called improper.

A self-contained exposition of this foundational material on affine hypersurfaces may be found in Nomizu [6]. This source includes references to the original work of Blaschke [1] and others. 
Specializing now to the situation in which we will be interested in this paper, the following is immediate from (5) and (6).

Lemma 1. Let $f: M^{n} \rightarrow R^{n+1}$ be an affine hypersphere so that $S=\lambda I$. Suppose that the affine metric $h$ is of constant curvature $a$. Then

$$
J=a-\lambda
$$

and is therefore constant. Also

$$
\left[K_{X}, K_{Y}\right] Z=(\lambda-a)(h(Y, Z) X-h(X, Z) Y) .
$$

\section{EXAMPLES AND THE MAIN THEOREM}

We now introduce the three examples we wish to characterize. They are determined by three algebraic equations of degree 4 . The solution to equations (I) and (II) have more than one component. To avoid confusion, we take the component containing a point with positive coordinates as our target hypersurface. All three examples are proper affine 3-spheres and each is flat in the affine metric. Hence the Pick invariant is nonzero. The affine metric is positive definite in (I) and Lorentz in (II) and (III). The equations are:

$$
\begin{gathered}
x_{1} x_{2} x_{3} x_{4}=1, \\
\left(x_{1}^{2}+x_{2}^{2}\right)\left(x_{3}^{2}-x_{4}^{2}\right)=1, \\
\left(x_{1}^{2}+x_{2}^{2}\right)\left(x_{3}^{2}+x_{4}^{2}\right)=1 .
\end{gathered}
$$

Our main theorem now characterizes these hypersurfaces as follows:

Theorem. Let $f: M^{3} \rightarrow R^{4}$ be an affine hypersphere with nonzero Pick invariant and constant curvature $a$ in the affine metric $h$. Then $a=0$ and $f$ embeds $M$ onto an open subset of one of the examples (I)-(III) modulo an affine transformation of $R^{4}$.

Yu [9] characterized (I) by assuming $h$ positive definite, proving that $a=$ 0 , and then appealing to a general theorem of Li [4]. We apply the same techniques to the indefinite case but then find (II) and (III) directly by solving the differential equations. Our method can also be adapted to yield a direct proof of Yu's result.

If the condition on $J$ is omitted, the quadric hypersurfaces may be added to the list. In the definite case, these are the only additional possibilities, as $\mathrm{Yu}$ has noted. However, we cannot draw this conclusion in the indefinite case.

\section{The Laplacian of the Pick InVariant}

In this section, we develop some general formulas for the Laplacian of the Pick invariant of an affine hypersurface. For $n=2$, these results may be found in Blaschke [1, p. 211].

Lemma 2. Let $f: M^{n} \rightarrow R^{n+1}$ be a (nondegenerate) affine hypersurface with cubic form $C$, affine metric $h$, and Pick invariant $J$. Then 


$$
n(n-1) \Delta J=\frac{1}{2} h\left(\Delta^{\prime} C, C\right)+\frac{1}{2} h(\widehat{\nabla} C, \hat{\nabla} C),
$$

where $\Delta^{\prime} C$ is the $(0,3)$ tensor field obtained by taking the trace of the second covariant derivative of $C$. In terms of an orthonormal basis, $\left\{e_{i}\right\}$,

$$
\Delta^{\prime} C=\sum\left(\widehat{\nabla}^{2} C\right)\left(; e_{i} ; e_{i}\right) h\left(e_{i}, e_{i}\right) .
$$

Proof. We start from the definition of the second covariant derivative

$$
\left(\widehat{\nabla}^{2} J\right)(; Y ; X) \equiv \widehat{\nabla}_{X} \widehat{\nabla}_{Y} J-\widehat{\nabla}_{\widehat{\nabla}_{X} Y} J
$$

Noting that

$$
4 n(n-1) J=h(C, C)
$$

we get

$$
\begin{aligned}
4 n(n-1)\left(\widehat{\nabla}^{2} J\right)(; Y ; X) & =\widehat{\nabla}_{X}\left(2 h\left(\hat{\nabla}_{Y} C, C\right)\right)-2 h\left(\hat{\nabla}_{\widehat{\nabla}_{X} Y} C, C\right) \\
& =2 h\left(\left(\widehat{\nabla}^{2} C\right)(; Y ; X), C\right)+2 h\left(\widehat{\nabla}_{X} C, \widehat{\nabla}_{Y} C\right) .
\end{aligned}
$$

Taking the trace of both sides yields the desired formula.

Lemma 3. Let $f: M^{n} \rightarrow R^{n+1}$ be an affine hypersphere whose affine metric $h$ has constant curvature $a$. Then

$$
\Delta^{\prime} C=a(n+1) C .
$$

Proof. Let us use the following notation for the second covariant derivative of $C$ :

$$
\left(\widehat{\nabla}^{2} C\right)(U, V, W, Y, X) \equiv\left(\widehat{\nabla}_{X} \widehat{\nabla}_{Y} C-\widehat{\nabla}_{\hat{\nabla}_{X} Y} C\right)(U, V, W) .
$$

It is a fact due to Bokan, Nomizu, and Simon [2] that, for an affine hypersurface, $\hat{\nabla} C$ is symmetric in its four arguments if and only if the hypersurface is an affine hypersphere. It follows that the $(0,5)$ tensor $\hat{\nabla}^{2} C$ is symmetric in its first four arguments. Permuting the last two arguments allows us to introduce a curvature term. In other words,

$$
\begin{aligned}
& \left(\widehat{\nabla}^{2} C\right)(U, V, W, Y, X)=\left(\widehat{\nabla}^{2} C\right)(Y, V, W, U, X) \\
& \quad=\left(\widehat{\nabla}^{2} C\right)(Y, V, W, X, U)+(\widehat{R}(X, U) \cdot C)(Y, V, W),
\end{aligned}
$$

where $\widehat{R}(X, U)$ acts as a derivation on the algebra of tensor fields of $M$. Since $\widehat{R}$ has constant curvature $a$, we can compute

$$
\begin{aligned}
(\widehat{R}(X, U) \cdot C)(Y, V, W) \\
=-C(\widehat{R}(X, U) Y, V, W)-C(\widehat{R}(X, U) V, Y, W) \\
\quad-C(\widehat{R}(X, U) W, Y, V) \\
=-a(h(U, Y) C(X, V, W)-h(X, Y) C(U, V, W) \\
\quad+h(U, V) C(X, Y, W)-h(X, V) C(U, Y, W) \\
\quad+h(U, W) C(X, Y, V)-h(X, W) C(U, Y, V)) .
\end{aligned}
$$

We now must take the trace. This can be done using indices in a tedious but 
straightforward way. However, it is perhaps more instructive to give an "invariant" calculation and we shall do this. We look at the curvature term first. It is possible to isolate its dependence on $Y$ by writing

$$
(\widehat{R}(X, U) \cdot C)(Y, V, W)=h(\Gamma(U, V, W, X), Y),
$$

and then we need only compute the trace of the mapping

$$
\{X \rightarrow \Gamma(U, V, W, X)\} .
$$

Now

$$
\begin{aligned}
\Gamma(U, V, W, X)=-a( & C(X, V, W) U-C(U, V, W) X-2 h(U, V) K_{W} X \\
& \left.+2 h(X, V) K_{U} W-2 h(U, W) K_{V} X+2 h(X, W) K_{U} V\right)
\end{aligned}
$$

from which the trace is

$$
\begin{aligned}
& -a\left(C(U, V, W)-n C(U, V, W)-0+2 h\left(K_{U} W, V\right)-0+2 h\left(K_{U} V, W\right)\right) \\
& \quad=a(n+1) C(U, V, W) .
\end{aligned}
$$

We now look at the other term. First define $T$ by

$$
\left(\widehat{\nabla}_{Y} C\right)(X, V, W)=h(T(X, V, W), Y) .
$$

Then it is not difficult to check that

$$
\left(\widehat{\nabla}^{2} C\right)(X, V, W, Y, U)=h\left(\left(\widehat{\nabla}_{U} T\right)(X, V, W), Y\right) .
$$

We complete the proof by showing that the trace of the map

$$
\left\{X \rightarrow\left(\widehat{\nabla}_{U} T\right)(X, V, W)\right\}
$$

is 0 . To this end we note that

$$
\begin{aligned}
\left(\hat{\nabla}_{U} T\right)(X, V, W)= & \hat{\nabla}_{U}(T(X, V, W))-T\left(\widehat{\nabla}_{U} X, V, W\right) \\
& -T\left(X, \widehat{\nabla}_{U} V, W\right)-T\left(X, V, \widehat{\nabla}_{U} W\right) .
\end{aligned}
$$

Using (4) and the fact that $\hat{\nabla}_{X}$ acts as a derivation and commutes with contractions, one can verify that for any $X, Y$, and $Z$

$$
T(X, Y, Z)=-2\left(\widehat{\nabla}_{Y} K\right)_{Z} X \text {. }
$$

On the other hand,

$$
\begin{gathered}
\left(\widehat{\nabla}_{Y} K\right)_{Z}=\widehat{\nabla}_{Y}\left(K_{Z}\right)-K_{\widehat{\nabla}_{Y} Z}, \\
\operatorname{trace} \widehat{\nabla}_{Y}\left(K_{Z}\right)=\widehat{\nabla}_{Y}\left(\operatorname{trace} K_{Z}\right)=0=\operatorname{trace} K_{\widehat{\nabla}_{Y} Z}
\end{gathered}
$$

by the apolarity condition. Thus, for any $Y$ and $Z$,

$$
\operatorname{trace}\{X \rightarrow T(X, Y, Z)\}=0
$$

and the last two terms of (9) each contribute 0 to the trace. Furthermore, writing

$$
\widetilde{T}(X)=T(X, V, W)
$$

we have

$$
\begin{aligned}
0 & =\widehat{\nabla}_{U}(\operatorname{trace} \widetilde{T})=\operatorname{trace}\left(\widehat{\nabla}_{U} \widetilde{T}\right)=\operatorname{trace}\left\{X \rightarrow\left(\widehat{\nabla}_{U} \widetilde{T}\right)(X)\right\} \\
& =\operatorname{trace}\left\{X \rightarrow \widehat{\nabla}_{U}(\widetilde{T}(X))\right\}-\operatorname{trace}\left\{X \rightarrow \widetilde{T}\left(\widehat{\nabla}_{U} X\right)\right\} \\
& =\operatorname{trace}\left\{X \rightarrow \widehat{\nabla}_{U}(T(X, V, W))\right\}-\operatorname{trace}\left\{X \rightarrow T\left(\widehat{\nabla}_{U} X, V, W\right)\right\}
\end{aligned}
$$


Thus the first two terms of (9) taken together also contribute 0 to the trace. This completes the proof of Lemma 3.

Corollary. Under the conditions of Lemma 3 , if $h$ is positive definite and $a \geq 0$, either $C$ must vanish identically or a must be 0 and $\widehat{\nabla} C$ must be zero.

Proof. The Pick invariant $J$ is constant. Substituting the result of Lemma 3 in (7) yields

$$
a(n+1) h(C, C)+h(\widehat{\nabla} C, \widehat{\nabla} C)=0,
$$

from which the result follows.

\section{COMPONENTS OF THE CUBIC FORM}

Notation. If $\left\{e_{i}\right\}$ is an orthonormal basis, the coefficients of the brackets of the various $K_{e_{i}}$ are defined by

$$
\left[K_{e_{i}}, K_{e_{j}}\right] e_{m}=\sum\left[K_{e_{t}}, K_{e_{j}}\right]_{k m} e_{k}
$$

(summation over $k$ ). $C\left(e_{i}, e_{j}, e_{k}\right)$ will be written $C_{i j k}$ and $\left(\widehat{\nabla}_{e_{m}} C\right)\left(e_{i}, e_{j}, e_{k}\right)$ will be written $C_{i j k m}$. This last notation is used only in Lemma 9.

In this section we list the basic identities satisfied by the components of the cubic form of an affine hypersurface in $R^{4}$ for which the affine metric $h$ is Lorentz. We use an orthonormal basis $\left\{e_{1}, e_{2}, e_{3}\right\}$ with $e_{1}$ timelike, and $e_{2}$ and $e_{3}$ spacelike. From the relationship between $C$ and $K$ in (4) we get the following:

$$
\begin{aligned}
4\left[K_{e_{1}}, K_{e_{2}}\right]_{12}= & \left(C_{111}+C_{122}\right) C_{122}-\left(C_{112}+C_{222}\right) C_{112} \\
& -C_{113} C_{223}+C_{123}^{2}, \\
4\left[K_{e_{1}}, K_{e_{3}}\right]_{13}= & \left(C_{111}+C_{133}\right) C_{133}-\left(C_{113}+C_{333}\right) C_{113} \\
& -C_{112} C_{233}+C_{123}^{2}, \\
4\left[K_{e_{2}}, K_{e_{3}}\right]_{23}= & \left(C_{222}-C_{233}\right) C_{233}-\left(C_{223}-C_{333}\right) C_{223} \\
& -C_{122} C_{133}+C_{123}^{2}, \\
4\left[K_{e_{1}}, K_{e_{2}}\right]_{13}= & \left(C_{111}+C_{133}\right) C_{123}-\left(C_{112}+C_{233}\right) C_{113} \\
& -C_{112} C_{223}+C_{123} C_{122}, \\
4\left[K_{e_{1}}, K_{e_{2}}\right]_{23}= & \left(C_{122}-C_{133}\right) C_{223}-\left(C_{222}-C_{233}\right) C_{123} \\
& -C_{112} C_{123}+C_{113} C_{122}, \\
4\left[K_{e_{2}}, K_{e_{3}}\right]_{13}= & \left(C_{112}+C_{233}\right) C_{133}-\left(C_{113}+C_{333}\right) C_{123} \\
& -C_{122} C_{233}+C_{223} C_{123}, \\
4\left[K_{e_{2}}, K_{e_{3}}\right]_{12}= & \left(C_{112}+C_{222}\right) C_{123}-\left(C_{113}+C_{223}\right) C_{122} \\
& -C_{233} C_{123}+C_{133} C_{223}, \\
4\left[K_{e_{1}}, K_{e_{3}}\right]_{12}= & \left(C_{111}+C_{122}\right) C_{123}-\left(C_{113}+C_{223}\right) C_{112} \\
& -C_{113} C_{233}+C_{123} C_{133},
\end{aligned}
$$




$$
\begin{aligned}
4\left[K_{e_{1}}, K_{e_{3}}\right]_{23}= & \left(C_{122}-C_{133}\right) C_{233}+\left(C_{333}-C_{223}\right) C_{123} \\
& -C_{112} C_{133}+C_{113} C_{123} .
\end{aligned}
$$

In the case of an affine hypersphere with constant affine curvature, Lemma $1,\left(6^{\prime}\right)$ may be used to turn the above formulas into a set of identities for the coefficients of $C$. We state these identities, along with the apolarity condition, as follows:

Lemma 4. Let $f: M^{3} \rightarrow R^{4}$ be an affine hypersphere so that $S=\lambda I$. Suppose that the affine metric $h$ is a Lorentz metric of constant curvature $a$. Then

(i) The right sides of equations (10.1)-(10.3) are all equal to $\lambda-a$. Furthermore, the right sides of (11.1)-(11.6) are all equal to zero.

(ii) The apolarity condition takes the form

$$
\begin{aligned}
& C_{111}=C_{122}+C_{133}, \\
& C_{112}=C_{222}+C_{233}, \\
& C_{113}=C_{223}+C_{333} .
\end{aligned}
$$

\section{Computation of $\Delta J$}

The purpose of this section is to study hypersurfaces satisfying the hypotheses of the main theorem and, by a suitable choice of orthonormal basis of each tangent space, make the form of the $C_{i j k}$ as simple as possible, eventually showing that $\widehat{\nabla} C=0$. Since the development is rather lengthy, we will break it up into a sequence of lemmas.

Lemma 5. Let $f: M^{3} \rightarrow R^{4}$ be an affine hypersphere whose affine metric $h$ is a Lorentz metric of constant curvature with nonzero Pick invariant. At any point of $M$, the tangent space has an orthonormal basis such that two of the three components of the form $C_{i i i}$ are zero.

Proof. Denote by $V$ the tangent space at the point in question. Let

$$
B=\{x \in V \mid h(x, x)=-1\}
$$

and let $B^{+}$be a component of $B$ on which $g(x)=C(x, x, x)$ achieves at least one nonnegative value. If the value 0 is achieved, let $e_{1}$ be a point where this occurs. Then, on the spacelike plane orthogonal to $e_{1}$, we may choose an orthonormal basis $\left\{e_{2}, e_{3}\right\}$. If $C_{333}=0$ we are finished. Otherwise, let

$$
\bar{e}_{2}=\cos \theta e_{2}+\sin \theta e_{3}, \quad \bar{e}_{3}=-\sin \theta e_{2}+\cos \theta e_{3}
$$

and note that

$$
C\left(\bar{e}_{3}, \bar{e}_{3}, \bar{e}_{3}\right)=-\sin ^{3} \theta\left(C_{222}-3 C_{223} \cot \theta+3 C_{233} \cot ^{2} \theta-C_{333} \cot ^{3} \theta\right)
$$

so $\theta$ may be chosen to make $C\left(\bar{e}_{3}, \bar{e}_{3}, \bar{e}_{3}\right)=0$.

Now assume that $g(x)>0$ on $B^{+}$. Let $e_{1}$ be a point where the minimum is achieved (see Lemma 5a below). As in the previous paragraph, choose $\left\{e_{2}, e_{3}\right\}$ so that $C_{333}=0$. For $X$ tangent to $B^{+}$at $e_{1}, X g=3 C(X, x, x)$ at $x=e_{1}$. Setting $X=e_{2}$ and $X=e_{3}$ in turn yields that $C_{112}$ and $C_{113}$ both vanish.

We now show that $C_{222}$ must vanish. Assume not. Since $C_{333}, C_{113}, C_{112}$ are all zero, the apolarity conditions reduce to

$$
C_{111}=C_{122}+C_{133}, \quad C_{222}=-C_{233}, \quad C_{223}=0 .
$$


Thus formulas (10.1)-(10.3) yield

$$
\left(C_{111}+C_{122}\right) C_{122}=\left(C_{111}+C_{133}\right) C_{133}=-2 C_{222}^{2}-C_{122} C_{133},
$$

while formulas $(11.1)-(11.6)$ give (twice each)

$$
C_{111} C_{123}=0, \quad C_{222} C_{123}=0, \quad\left(C_{122}-C_{133}\right) C_{222}=0 .
$$

Using the fact that neither $C_{111}$ nor $C_{222}$ can be zero, we see that $C_{111}=$ $2 C_{122}=2 C_{133}$ and substituting this in (13) gives

$$
C_{111}^{2}+2 C_{222}^{2}=0 \text {, }
$$

a contradiction. This completes the proof of Lemma 5.

In Lemma 5, we deferred the proof that $g$ achieves a minimum on $B^{+}$, since it is somewhat technical and would make the proof of Lemma 5 too long. We now present this as Lemma 5 a.

Lemma 5a. Assume the hypotheses of Lemma 5. In the tangent space at a fixed point of $M$, let $B^{+}$be a component of $B$ (the unit timelike vectors) on which $g(x)=C(x, x, x)$ is positive. Then $g$ achieves a minimum on $B^{+}$.

Proof. Consider an orthonormal basis $\left\{e_{1}, e_{2}, e_{3}\right\}$ with $e_{1} \in B^{+}$, and $e_{2}$ and $e_{3}$ spacelike. In terms of this basis, a typical point of $B^{+}$may be expressed as

$$
x=(\cosh u, \cos \theta \sinh u, \sin \theta \sinh u)
$$

for $0 \leq \theta \leq 2 \pi, u \geq 0$, and we may write

$$
g(x)=C_{111}\left(\cosh ^{3} u+p \cosh ^{2} u \sinh u+q \cosh u \sinh ^{2} u+r \sinh ^{3} u\right),
$$

where $p, q$, and $r$ are analytic functions of $\theta$. Note that $C_{111}=g\left(e_{1}\right)>0$. Set $t=\cosh u$ and $s=\sinh u=\left(t^{2}-1\right)^{1 / 2}$ for $u \geq 0$. For a fixed value of $\theta$, consider the positive function

$$
h(t)=t^{3}+p t^{2} s+q t s^{2}+r s^{3}
$$

which can be simplified to

$$
h(t)=(1+p+q+r) t^{3}-\frac{1}{2}(p+2 q+3 r) t+\rho(t),
$$

where the remainder $\rho$ is given by

$$
\rho(t)=\frac{-p}{2 t(1+s / t)^{2}}+\frac{r}{s+t}\left(1-\frac{1}{2(1+s / t)}\right) .
$$

Since $t \geq 1$, we have

$$
|\rho(t)| \leq \frac{1}{2}(|p|+2|r|)
$$

and thus $|\rho(t)| \leq \mu$, where $2 \mu$ is the maximum of $|p|+2|r|$ over $\theta$.

We claim that $h(t)$ goes to $\infty$ with $t$ and does so in a sufficiently uniform way to force $g(x)$ to have a minimum. Specifically, we will show that for any positive number $\alpha$ there is a number $t_{0}$ independent of $\theta$ such that $h(t) \geq \alpha$ for all $t \geq t_{0}$. This will allow us to choose a closed disk in $B^{+}$, centered at $e_{1}$ outside of which $g(x)>2 g\left(e_{1}\right)$. The minimum of $g$ on this disk will be the global minimum of $g$.

In order to establish the required bounds, we prove the following claim. Note we need to use the fact that the Pick invariant is nonzero. 
Claim. In the notation introduced above, we have $1+p+q+r \geq 0$ and if $1+p+q+r=0$, then $p+2 q+3 r<0$.

Proof of claim. We are interested in the behavior of $g(x)$ along an arbitrary geodesic of $B^{+}$with initial point $e_{1}$. For the sake of this calculation, no generality is lost in assuming that $\theta=0$. The limit as $t$ goes to $\infty$ of $h(t) / t^{3}$ is $1+p+q+r$ so this quantity is nonnegative. If it happens to be zero, then taking the limit of $h(t) / t$ shows $-(p+2 q+3 r)$ to be nonnegative. Suppose now that both $1+p+q+r$ and $p+2 q+3 r$ are zero. Then

$$
0=C_{111}(1+p+q+r)=C_{111}+3 C_{112}+3 C_{122}+C_{222}
$$

and

$$
0=C_{111}(p+2 q+3 r)=3 C_{112}+6 C_{122}+3 C_{222} .
$$

Writing $\tau=C_{111}$ and $\sigma=C_{222}$, we get

$$
3 C_{122}=\tau-2 \sigma, \quad 3 C_{112}=\sigma-2 \tau,
$$

and the apolarity condition gives

$$
3 C_{133}=2(\tau+\sigma)=-3 C_{233} .
$$

Substituting in (10.1)-(10.3) yields $C_{333}=0$ and $\tau+\sigma=0$, and hence $C_{223}=$ $C_{113}$ by apolarity. From (11.1), we get $C_{113}=-C_{123}$ and substituting back in (10.1) gives 0 . Since the Pick invariant $J \neq 0$, we have a contradiction. (It can be checked that if $J$ were allowed to be zero, any $C$ satisfying the identities we have derived in the present proof would satisfy all the identities summarized in Lemma 4.)

The claim having been established, we return to the proof of Lemma 5a. Note that the analytic function $1+p+q+r$ has only finitely many zeros on the unit circle, unless it is identically zero. Let $4 \delta$ be the smallest of the values taken by $-(p+2 q+3 r)$ on the zero set. If there are finitely many zeros, surround each by a closed interval on which $-(p+2 q+3 r) \geq 2 \delta$. Then

$$
(1+p+q+r) t^{2}-\frac{1}{2}(p+2 q+3 r) \geq \delta
$$

on the union $\mathscr{A}$ of these intervals. This holds for all $t \geq 1$. Now let $\beta$ be the minimum of $(1+p+q+r)$ on the closure $\mathscr{B}$ of the complement of $\mathscr{A}$. Let $2 \gamma$ be the maximum of $|p+2 q+3 r|$ on $\mathscr{B}$. Then

$$
(1+p+q+r) t^{2}-\frac{1}{2}(p+2 q+3 r) \geq \beta t^{2}-\gamma \geq \delta
$$

on $\mathscr{B}$ provided that $t^{2} \geq(\delta+\gamma) / \beta$. Choosing $t_{0}$ to be the maximum of $(\delta+\gamma) / \beta,(\mu+\alpha) / \delta$, and 1 (or in case $1+p+q+r \equiv 0, t_{0}=(\mu+\alpha) / \delta$ ), we can conclude that

$$
h(t) \geq \delta t-\mu \geq \alpha
$$

for $t \geq t_{0}$. Note that $t_{0}$ is independent of $\theta$. This completes the proof of Lemma 5a.

Lemma 6. Let $f: M^{3} \rightarrow R^{4}$ be an affine hypersphere whose affine metric $h$ is a Lorentz metric of constant curvature with nonzero Pick invariant. At any point 
of $M$ the tangent space has an orthonormal basis such that all three components of the form $C_{i i i}$ are zero.

Proof. Using the result of Lemma 5, there are two cases to consider. First assume that $C_{333}$ and $C_{222}$ vanish. Since $h$ is Lorentz, the apolarity conditions are

$$
C_{111}=C_{122}+C_{133}, \quad C_{112}=C_{233}, \quad C_{113}=C_{223} .
$$

We use these simplifications and (10.2)-(10.3) to get

$$
\begin{aligned}
& C_{111} C_{133}+C_{133}^{2}-C_{113}^{2}-C_{112}^{2}+C_{123}^{2} \\
& \quad=-C_{112}^{2}-C_{113}^{2}-C_{122} C_{133}+C_{123}^{2} .
\end{aligned}
$$

That is,

$$
C_{133}\left(C_{111}+C_{122}+C_{133}\right)=0
$$

in other words, $C_{133} C_{111}=0$. By symmetry, we also have $C_{122} C_{111}=0$. Adding these equations, we have $C_{111}=0$, as required. On the other hand, if we assume that $C_{333}$ and $C_{111}$ vanish (where $e_{1}$ is timelike), the apolarity conditions become

$$
C_{122}=-C_{133}, \quad C_{112}=C_{222}+C_{233}, \quad C_{113}=C_{223} .
$$

Equating (10.1) and (10.2) gives $C_{112} C_{222}=0$ while (10.2) and (10.3) give

$$
C_{233}\left(C_{222}+C_{112}-C_{233}\right)=0 \text {, }
$$

that is, $C_{222} C_{233}=0$. Subtracting our two conditions gives $C_{222}\left(C_{112}-C_{233}\right)=$ 0 which means that $C_{222}=0$.

Lemma 7. Let $f: M^{3} \rightarrow R^{4}$ be an affine hypersphere with $S=\lambda I$ whose affine metric $h$ is a Lorentz metric of constant curvature $a$ and nonzero Pick invariant. Then at any point of $M$ the tangent space has an orthonormal basis such that all components of $C$ vanish except (i) $C_{112}=C_{233}$ in the case $\lambda-a<0$; (ii) $C_{123}$ in the case $\lambda-a>0$.

Proof. Start with the orthonormal basis constructed in Lemma 6. The apolarity conditions take on the simple form

$$
C_{122}=-C_{133}, \quad C_{112}=C_{233}, \quad C_{113}=C_{223},
$$

and formulas (11.1)-(11.6) now reduce to

$$
C_{112} C_{113}=C_{112} C_{122}=C_{113} C_{122}=0 .
$$

First consider the case where $C_{112} \neq 0$. Then all components of $C$ are zero except $C_{112}=C_{233}$ and possibly $C_{123}$. We consider a translation (terminology chosen by analogy with that used in hyperbolic geometry, see [7, p. 165] of the form

$$
\bar{e}_{1}=\cosh \theta e_{1}+\sinh \theta e_{3}, \quad \bar{e}_{2}=e_{2}, \quad \bar{e}_{3}=\sinh \theta e_{1}+\cosh \theta e_{3} .
$$

Then

$$
\begin{aligned}
& C\left(\bar{e}_{1}, \bar{e}_{1}, \bar{e}_{2}\right)=\cosh 2 \theta C_{112}+\sinh 2 \theta C_{123}, \\
& C\left(\bar{e}_{1}, \bar{e}_{2}, \bar{e}_{3}\right)=\sinh 2 \theta C_{112}+\cosh 2 \theta C_{123} .
\end{aligned}
$$

In case (i), (10.1) gives $-C_{112}^{2}+C_{123}^{2}<0$ and $\theta$ may be chosen to make the new $C_{123}$ vanish while no new nonzero components are introduced. On the other hand, if case (ii) obtains, we choose $\theta$ to make $C_{112}$ vanish. 
If $C_{113} \neq 0$ we merely switch the $e_{2}$ and $e_{3}$ to reach the same conclusion. However, if both $C_{112}$ and $C_{113}$ vanish, we use (10.1) to get

$$
C_{122}^{2}+C_{123}^{2}=\lambda-a \text {. }
$$

Thus $\lambda-a>0$ and we may choose a rotation of $\left\{e_{2}, e_{3}\right\}$ to make $C_{122}=0$ so $C_{123}$ is the only nonzero component.

Lemma 8. Let $M^{n}$ be a pseudo-Riemannian manifold. Any orthonormal basis in the tangent space at a point $x_{0}$ can be extended to a local orthonormal frame field whose covariant derivatives with respect to the Levi-Civita connection all vanish at $x_{0}$.

Proof. Extend the basis vectors by parallel translation along each geodesic emanating from $x_{0}$.

Lemma 9. Let $f: M^{3} \rightarrow R^{4}$ be an affine hypersphere whose affine metric $h$ is a Lorentz metric of constant curvature with nonzero Pick invariant. Let $C$ be the cubic form. Then $\widehat{\nabla} C=0$.

Proof. Choose any point $x_{0}$ of $M$. Depending upon the sign of the Pick invariant, choose an orthonormal basis for the tangent space to $M$ at $x_{0}$ as in Lemma 7 and extend it locally as in Lemma 8. The identities associated with (10.1)-(10.3) and (11.1)-(11.6) are now valid locally. Thus we differentiate them and evaluate at $x_{0}$. We separate the two cases and number each condition to correspond to that of the identity from which it was derived. For example, $\left(10.1^{\prime}\right)$ is obtained by differentiating $(10.1)$ and evaluating at $x_{0}$. In the following lines, the prime stands for differentiation with respect to any direction, in particular the orthonormal basis at $x_{0}$. Also, because the covariant derivatives of the basis vectors vanish at $x_{0}$, we have

$$
C_{i j k m}=\left(\widehat{\nabla}_{e_{m}} C\right)\left(e_{i}, e_{j}, e_{k}\right)=\hat{\nabla}_{e_{m}}\left(C\left(e_{i}, e_{j}, e_{k}\right)\right)
$$

at $x_{0}$.

Case (i): $\lambda-a<0$.

$$
\begin{aligned}
2 C_{112}^{\prime}+C_{222}^{\prime} & =0, \\
C_{112}^{\prime}+C_{233}^{\prime} & =0, \\
2 C_{113}^{\prime}+C_{223}^{\prime} & =0, \\
2 C_{133}^{\prime}-C_{122}^{\prime} & =0 .
\end{aligned}
$$

Combining these with $(12.1)-(12.3)$ it is easy to check that

$$
\begin{gathered}
C_{112}^{\prime}=C_{222}^{\prime}=C_{233}^{\prime}=0, \\
3 C_{223}^{\prime}=-6 C_{113}^{\prime}=-2 C_{333}^{\prime}, \\
3 C_{122}^{\prime}=6 C_{133}^{\prime}=2 C_{111}^{\prime} .
\end{gathered}
$$

We want to show that all terms of the form $C_{i j k m}$ vanish. Using the identities just derived and the symmetry of the $C_{i j k m}$, we get

$$
\begin{gathered}
C_{1111}=3 C_{1331}=3 C_{1133}=-\frac{3}{2} C_{2233}=-\frac{3}{2} C_{2332}=0, \quad C_{1112}=C_{1121}=0, \\
C_{1113}=C_{1131}=-\frac{1}{2} C_{2231}=-\frac{1}{2} C_{1223}=-C_{1333}=-\frac{1}{3} C_{1113},
\end{gathered}
$$


so that $C_{1113}=0$.

$$
C_{1233}=C_{1332}=\frac{1}{2} C_{1222}=\frac{1}{2} C_{2221}=0 .
$$

All other terms of the form $C_{i j k m}$ can be shown to be zero in one step, as the reader can readily verify.

Case (ii): $\lambda-a>0$.

$$
\begin{aligned}
C_{123}^{\prime} & =0, \\
C_{111}^{\prime}+C_{122}^{\prime}+C_{133}^{\prime} & =0, \\
C_{112}^{\prime}+C_{222}^{\prime}-C_{233}^{\prime} & =0, \\
C_{113}^{\prime}-C_{223}^{\prime}+C_{333}^{\prime} & =0 .
\end{aligned}
$$

Equations $\left(11.1^{\prime}\right)-\left(11.3^{\prime}\right)$ along with the apolarity identities merely say that $C_{i i i}^{\prime}=0$ for all $i$. To complete the proof in this case, just note that every term of the form $C_{i i i j}$ vanishes so that if any index occurs three or four times, the term vanishes. On the other hand, if exactly one index occurs twice, the term is of the form $C_{123 i}$ and therefore vanishes. Finally,

$$
C_{1221}=-C_{1331}=-C_{1133}=-C_{2233}=-C_{2332}=-C_{1122}=-C_{1221} \text {, }
$$

so the case of two repeated indices is also taken care of. We conclude that $\hat{\nabla} C$ vanishes at $x_{0}$. But $x_{0}$ was an arbitrary point of $M$ so $\widehat{\nabla} C=0$ everywhere, as claimed.

Proposition. Let $f: M^{3} \rightarrow R^{4}$ be an affine hypersphere with $S=\lambda I$ whose affine metric $h$ is a Lorentz metric of constant curvature a with nonzero Pick invariant $J$. Then $a=0$.

Proof. Applying Lemmas 9, 2, and 3 and using the fact that $J$ is constant gives us that

$$
0=\Delta J=\frac{1}{2} a(n+1) h(C, C)
$$

at $x_{0}$. Now $h(C, C)$ is the sum of terms of the form

$$
C_{i j k}^{2} h\left(e_{i}, e_{i}\right) h\left(e_{j}, e_{j}\right) h\left(e_{k}, e_{k}\right) .
$$

We note that evaluation of $h(C, C)$ involves only one term $C_{123}$ in Case (ii) and only two terms with the same sign in Case (i) since $C_{112}^{2}$ and $C_{233}^{2}$ both have an even number of indices equal to 1 . Thus, in either case, we must have $a=0$.

\section{THE MAIN THEOREM}

Theorem. Let $f: M^{3} \rightarrow R^{4}$ be an affine hypersphere whose affine metric $h$ is a Lorentz metric of constant curvature. Assume that the (constant) Pick invariant is not zero. Then $M$ is related by an affine transformation to an open subset of

$$
\left(x_{1}^{2}+x_{2}^{2}\right)\left(x_{3}^{2}-x_{4}^{2}\right)=1
$$

or

$$
\left(x_{1}^{2}+x_{2}^{2}\right)\left(x_{3}^{2}+x_{4}^{2}\right)=1 .
$$

Proof. Since $a=0$, the local orthonormal frame of Lemma 8 may be taken to consist of coordinate vectors $\frac{\partial}{\partial u_{l}}$. Because $S=\lambda I$, we have $\xi_{i}=-\lambda f_{i}$, 
where the subscript $i$ indicates differentiation with respect to $u_{i}$. Thus there is a constant vector $E \in R^{4}$ such that $\xi+\lambda f=E$. There is no loss of generality in taking $E$ to be the origin.

We first consider Case (i). Set $b=\frac{1}{2} C_{112}$ so that $\lambda=-b^{2}$. Then using (1), (3), and (4), we get

$$
\begin{gathered}
f_{11}=-b f_{2}-b^{2} f, \quad f_{12}=b f_{1}, \quad f_{13}=0, \\
f_{22}=b^{2} f, \quad f_{23}=-b f_{3}, \quad f_{33}=-b f_{2}+b^{2} f .
\end{gathered}
$$

We now solve this system to find $f$. First note that

$$
\left(f_{2}-b f\right)_{1}=0, \quad\left(f_{2}+b f\right)_{3}=0,
$$

so that there are functions $\alpha\left(u_{2}, u_{3}\right)$ and $\beta\left(u_{1}, u_{2}\right)$ such that

$$
f_{2}-b f=\alpha, \quad f_{2}+b f=\beta .
$$

Thus

$$
2 b f=\beta-\alpha, \quad 2 f_{2}=\beta+\alpha .
$$

Using these equations to simplify (16) yields

$$
\beta_{11}=-2 b^{2} \beta, \quad \alpha_{33}=2 b^{2} \alpha
$$

from which we can write

$$
\begin{aligned}
& \alpha=A\left(u_{2}\right) \cosh \left(\sqrt{2} b u_{3}\right)+B\left(u_{2}\right) \sinh \left(\sqrt{2} b u_{3}\right), \\
& \beta=C\left(u_{2}\right) \cos \left(\sqrt{2} b u_{1}\right)+D\left(u_{2}\right) \sin \left(\sqrt{2} b u_{1}\right)
\end{aligned}
$$

for suitable vector functions $A, B, C$, and $D$. However, by substituting in the identity

$$
\alpha_{2}+\beta_{2}=2 f_{22}=2 b^{2} f=b(\beta-\alpha)
$$

we can extract the fact that

$$
A^{\prime}+b A=B^{\prime}+b B=C^{\prime}-b C=D^{\prime}-b D=0
$$

and thus conclude that

$$
\begin{aligned}
& \alpha=A_{0} e^{-b u_{2}} \cosh \left(\sqrt{2} b u_{3}\right)+B_{0} e^{-b u_{2}} \sinh \left(\sqrt{2} b u_{3}\right), \\
& \beta=C_{0} e^{b u_{2}} \cos \left(\sqrt{2} b u_{1}\right)+D_{0} e^{b u_{2}} \sin \left(\sqrt{2} b u_{1}\right),
\end{aligned}
$$

where $A_{0}, B_{0}, C_{0}$, and $D_{0}$ are constant vectors. These vectors must be linearly independent, otherwise $f(M)$ would lie in a hyperplane, contradicting nondegeneracy. Thus, modulo the linear transformation that takes these four vectors to the standard basis of $R^{4}$, our hypersurface is nothing but the surface

$$
\left(x_{1}^{2}+x_{2}^{2}\right)\left(x_{3}^{2}-x_{4}^{2}\right)=1
$$

parametrized by

$$
\begin{array}{ll}
x_{1}=e^{b u_{2}} \cos \left(\sqrt{2} b u_{1}\right), & x_{2}=e^{b u_{2}} \sin \left(\sqrt{2} b u_{1}\right), \\
x_{3}=e^{-b u_{2}} \cosh \left(\sqrt{2} b u_{3}\right), & x_{4}=e^{-b u_{2}} \sinh \left(\sqrt{2} b u_{3}\right) .
\end{array}
$$

We now look at Case (ii). Set $b=-\frac{1}{2} C_{123}$ so that $\lambda=b^{2}$. Then (1) takes the form

$$
\begin{array}{ll}
f_{11}=b^{2} f, \quad f_{12}=-b f_{3}, & f_{13}=-b f_{2}, \\
f_{22}=-b^{2} f, \quad f_{23}=b f_{1}, & f_{33}=-b^{2} f .
\end{array}
$$


Now make a coordinate transformation replacing $u_{2}$ and $u_{3}$ by $\left(u_{2}-u_{3}\right) / \sqrt{2}$ and $\left(u_{2}+u_{3}\right) / \sqrt{2}$, respectively. Then our equations become

$$
\begin{gathered}
f_{11}=b^{2} f, \quad f_{12}=b f_{2}, \quad f_{13}=-b f_{3}, \\
f_{22}=-b f_{1}-b^{2} f, \quad f_{23}=0, \quad f_{33}=b f_{1}-b^{2} f .
\end{gathered}
$$

By the same method as used in Case (i), we find that our hypersurface coincides up to an affine transformation with the surface

$$
\left(x_{1}^{2}+x_{2}^{2}\right)\left(x_{3}^{2}+x_{4}^{2}\right)=1
$$

parametrized by

$$
\begin{array}{ll}
x_{1}=e^{b u_{1}} \cos \left(\sqrt{2} b u_{2}\right), & x_{2}=e^{b u_{1}} \sin \left(\sqrt{2} b u_{2}\right), \\
x_{3}=e^{-b u_{1}} \cos \left(\sqrt{2} b u_{3}\right), & x_{4}=e^{-b u_{1}} \sin \left(\sqrt{2} b u_{3}\right) .
\end{array}
$$

Although this characterization is only local, a connectedness argument such as that used in [5] shows that for a given affine hypersurface $f$, the affine transformation that works for an arbitrary small open set of $M$ works for all of $M$, so that we may say that $f$ maps $M$ onto an open subset of the target space in question.

\section{FURTHER REMARKS}

We have shown that a 3-dimensional affine hypersphere with Lorentz affine metric of constant curvature and nonzero Pick invariant must be one of two specific hypersurfaces up to affine transformation. Is it possible that these two are themselves equivalent? The answer is negative as the following lemma shows.

Lemma 10. Let $C$ be a nonzero trilinear function on a three-dimensional Lorentz vector space. Suppose that with respect to some orthonormal basis, only the $C_{123}$ component of $C$ is nonzero. Then there is no orthonormal basis with $e_{1}$ timelike such that $C_{112}$ and $C_{233}$ are the only nonzero components.

Proof. Let $\left\{e_{i}\right\}$ and $\left\{\tilde{e}_{i}\right\}$ be the respective bases. Then we may choose numbers $\theta$ and $\phi$ such that the intermediate basis $\left\{\bar{e}_{i}\right\}$ defined by

$$
\overline{\bar{e}}_{1}=\cosh \theta e_{1}+\sinh \theta e_{2}, \quad \bar{e}_{2}=\sinh \theta e_{1}+\cosh \theta e_{2}, \quad \bar{e}_{3}=e_{3}
$$

satisfies

$$
\tilde{e}_{1}=\bar{e}_{1}, \quad \tilde{e}_{2}=\cos \phi \bar{e}_{2}+\sin \phi \bar{e}_{3}, \quad \tilde{e}_{3}=-\sin \phi \bar{e}_{2}+\cos \phi \bar{e}_{3} .
$$

If $\widetilde{C}_{i j k}$ and $\bar{C}_{i j k}$ denote the components of $C$ in terms of the respective bases, then $\widetilde{C}_{123}=\cos 2 \phi \bar{C}_{123}=\cos 2 \phi \cosh 2 \theta C_{123}$. Thus $\cos 2 \phi$ must be zero and hence $\sin 2 \phi \neq 0$. But

$$
\widetilde{C}_{113}=-\sin \phi \bar{C}_{112}+\cos \phi \bar{C}_{113}=\cos \phi \sinh 2 \theta C_{123}
$$

and

$$
\widetilde{C}_{112}=\sin \phi \bar{C}_{113}=\sin \phi \sinh 2 \theta C_{123} .
$$

Since $\bar{C}_{112} \neq 0$, we must have $\sinh 2 \theta \neq 0$. But $\widetilde{C}_{113}$ vanishes so $\cos \phi=0$. This contradicts the fact that $\sin 2 \phi \neq 0$ and completes the proof that our two Lorentz target surfaces are distinct. 


\section{BIBLIOGRAPHY}

1. W. Blaschke, Vorlesungen über Differentialgeometrie, Vol. II, Springer, Berlin, 1923.

2. N. Bokan, K. Nomizu, and U. Simon, Affine hypersurfaces with parallel cubic forms, Tôhoku Math. J. 42 (1990), 101-108.

3. S.-Y. Cheng and S.-T. Yau, Complete affine hypersurfaces. The completeness of affine metrics, Comm. Pure Appl. Math. 36 (1986), 839-866.

4. Li An-Min, Some theorems in affine differential geometry, (preprint), 1988.

5. M. A. Magid and P. J. Ryan, Flat affine spheres in $R^{3}$, Geom. Dedicata 33 (1990), 277-288.

6. K. Nomizu, Introduction to affine differential geometry, Part I, MPI Preprint 88-37.

7. P. J. Ryan, Euclidean and non-Euclidean geometry, an analytic approach, Cambridge Univ. Press, Cambridge, New York, Melbourne, 1986.

8. U. Simon, Local classification of two dimensional affine spheres with constant curvature metric, Preprint No. 229/1989, Technische Universität Berlin.

9. Yu Jian-Hui, Affine hyperspheres with constant sectional curvature in $A^{4}$, preprint, 1989.

Department of Mathematics, Wellesley College, Wellesley, Massachusetts 02181

Department of Mathematics and Statistics, McMaster University, Hamilton, ONTARIO, L85 4K1 CANADA 